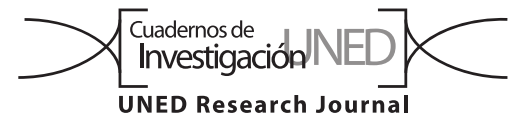

\title{
Producción y reproducción de vacas Holstein, Jersey y sus cruces en cinco localidades de Costa Rica
}

\author{
José Pablo Gonzáles Blanco' \& Rodolfo WingChing-Jones², \\ 1. Escuela de Zootecnia. Universidad de Costa Rica; espigajp@icloud.com \\ 2. Escuela de Zootecnia. Centro de Investigación en Nutrición Animal. Universidad de Costa Rica; rodolfo.wingching@ucr.ac.cr
}

Recibido 03-IV-2018 • Corregido 07-VI-2018 • Aceptado 14-VI-2018

\begin{abstract}
Production and reproduction of Holsteins, Jersey cows, and their breeding in five locations of Costa Rica. In Costa Rica $26 \%$ of the cattle is intented to the production of specialized milk, predominating the Jersey race, followed by Holstein, Guersey and Brown Swiss. We evaluated 1586 animal records in Heredia, Cartago, Coronado, San Carlos and Zarcero. The systems are characterized by the use of rotational grazing in African star (Cynodon nlemfuensis) or Kikuyo (Kikuyuocloa clandestina), complementing with agroindustrial byproducts, silages; and balanced feed with a minimum of $16 \%$ crude protein and $3000 \mathrm{kcal}$ ED / $\mathrm{kg}$. We use the updated records to make a database including open days (DA), interval between births (IEP), days in lactation and daily milk production of Holstein, Jersey, and their crosses in different proportions. Reproductive parameters, IEP and open days were higher in Holstein than in Jersey and F1 HxJ in 30,44 and 35,80; 20,29 and 25,86 days respectively. Daily, Jersey produces 1,77 and 1,3kg less milk than Holstein and F1 HxJ. There was no difference in Holstein and $\mathrm{F} 1 \mathrm{HxJ}$. The crossing of Holstein and Jersey is promising.
\end{abstract}

Keywords: daily milk production, animal reproduction, open days, calving interval, Bos taurus.
RESUMEN: En Costa Rica el $26 \%$ del ganado vacuno está destinado a la producción de leche especializada, predominando la raza Jersey, seguida de Holstein, Guersey y Pardo Suizo. Evaluamos 1586 registros de animales en Heredia, Cartago, Coronado, San Carlos y Zarcero. Los sistemas se caracterizan por el uso del pastoreo rotacional en estrella africana (Cynodon nlemfuensis) o kikuyo (Kikuyuocloa clandestina), complemenando con subproductos agroindustriales, ensilajes; y alimento balanceado con un mínimo de $16 \%$ de proteína cruda y de $3000 \mathrm{kcal} \mathrm{ED} / \mathrm{kg}$. Utilizamos los registros actualizados para hacer una base de datos incluyendo días abiertos (DA), intervalo entre partos (IEP), días en lactancia y producción de leche diaria de Holstein, Jersey, y sus cruces en diferentes proporciones. Los parámetros reproductivos, IEP y días abiertos, fueron mayores en Holstein que en Jersey y $\mathrm{F} 1 \mathrm{HxJ}$ en 30,44 y 35,$80 ; 20,29$ y 25,86 días respectivamente. Diariamente, la Jersey produce 1,77 y $1,3 \mathrm{~kg}$ menos leche que Holstein y F1 HxJ. No hubo diferencia en Holstein y F1 HxJ. El cruce de Holstein y Jersey es promisorio.

Palabras clave: Producción de leche, reproducción animal, periodo abierto, intervalo entre partos, Bos taurus.
Según el VI Censo Nacional Agropecuario del año 2014 (INEC 2015), el país cuenta con un total de 1278817 vacunos en 37171 fincas, donde el $25,6 \%$ de esta población se dedica a la producción de leche especializada. La información sobre composición racial de este grupo de animales en Costa Rica es escasa, imprecisa y desactualizada, pero resume que los animales de la raza Jersey son más abundantes, junto a los animales Holstein, Guersey y Pardo Suizo (Cordero-Solórzano, Vargas-Leiton, LeónRodríguez, Chacón-González \& Martínez-Pichardo, 2015).

Estos sistemas productivos, compuestos por la integración del recurso animal, suelo, cultivo, ambiente y manejo, afrontan retos todos los días para mantener su actividad (Vargas-Leitón, Solís-Guzmán, Sáenz-Segura \&
León-Hidalgo, 2015). Retos relacionados a la cuantificación de la huella del agua (Aamoun, 2015), huella de carbono (Rotz, Montes \& Chianese, 2010), el estrés calórico (Herbut \& Angrecka, 2017), presencia de enfermedades metabólicas (Bačić, Karadjole, Mačešić \& Karadjole, 2007) y aspectos nutricionales de los animales, con la meta de mantener la eficiencia del sistema y optimizar el uso de los insumos, la utilización de razas especializadas en producción de leche es una norma en estas empresas. Dicha situación genera cuestionamiento al contemplar que estos animales especializados, presentan problemas de adaptación a condiciones climáticas (McAllister, 2002), aumento en la consanguinidad (Vargas-Leitón \& Romero-Zúñiga, 2010); lo que reduce su productividad 
en el sistema, por aumento en el descarte de animales, por selección y disminución en la fertilidad (Buckley, Lopez-Villalobos \& Heins, 2014).

Como respuesta a esta situación, sumado a la dinámica del mercado de la leche, que reconoce el contenido de sólidos totales y no el volumen de leche (Vargas-Leitón \& Romero-Zúñiga, 2010), en estos sistemas productivos se implementa los cruces entre razas de animales productores, debido a que es una forma de incrementar la variación de los genes (VanRaden \& Sanders, 2003), lo que reduce la consanguinidad (Buckely et al., 2014), lo que permite obtener la ventaja de la heterosis o vigor híbrido (Echeverri, Salazar \& Parra, 2011).

La respuesta de los animales cruzados en parámetros ligados a la productividad, la salud, la reproducción y la rentabilidad en relación a las razas paternas o maternas, se resume en el trabajo realizado por Vásquez, Barrios \& Olivera (2015), donde indican estudios en Australia, Canadá, Chile, Costa Rica, Estados Unidos (Florida, Minnesota y Ohio), Honduras e Irlanda, donde resumen las mejoras de los animales en producción, reproducción, salud y rentabilidad. Por tal motivo, el objetivo de este trabajo fue comparar los índices zootécnicos, intervalo entre partos, días abiertos, días en lactancia promedio del hato, producción láctea y valor de urea en leche, de las razas Holstein, Jersey y sus cruces, en sistemas con pastoreo rotacional y suplementación en canoa, ubicados en cinco localidades productoras de Costa Rica.

\section{METODOLOGÍA}

Ubicación y caracterización de los sistemas: Los sistemas productivos que participaron en esta investigación se caracterizan por el uso de un modelo de pastoreo rotacional en forrajes tipo gramíneas de Estrella africana (Cynodon nlemfuensis) o Kikuyo (Kikuyuocloa clandestina). Además de suplementar en canoa con subproductos agroindustriales (banano, pulpa de cítricos deshidratada o cáscara de piña) y/o ensilajes producidos en las fincas de forrajes como maíz y sorgo, además del uso de un alimento balanceado con un mínimo de $16 \%$ de proteína cruda y de $3000 \mathrm{kcal}$ ED por kilogramo de alimento. Estas fincas tienen un manejo de lechería tipo especializada donde se ordeña dos veces al día con intervalos de 1212, 11-13 o 10-14 horas. Presentan registros actualizados sobre la producción de leche diaria, la reproducción de los animales expresada en el periodo abierto y el intervalo entre parto; y el manejo nutricional según la productividad del semoviente. Los sistemas de producción se ubicaron en las comunidades de Heredia, Cartago, Coronado, San Carlos y Zarcero. De estos sistemas se evaluaron 1586 registros completos, donde el aporte porcentual por cada localidad fue de 35,$88 ; 21,81 ; 4,72$; 31,$27 ;$ y $6,30 \%$ de los registros respectivamente.

Variables evaluadas y organización de la información: Se confeccionó una base de datos para las variables días abiertos (DA), intervalo entre partos (IEP), días en lactancia y producción de leche diaria. Esta información se extrajo de los registros productivos y reproductivos de los animales, digitados en el programa VAMPP Bovino ${ }^{\oplus}$ de cada finca. En el caso de los DA, el aporte porcentual para cada raza fue de 37,$36 ; 32,10$ y $30,54 \%$ datos para las razas Holstein, Jersey y los cruces de ambas en diferentes proporciones respectivamente. En cambio, para el IEP los porcentajes fueron 35,$71 ; 33,90$ y $30,39 \%$ respectivamente. Al analizar la variable días en lactancia se registró un aporte porcentual de $37,36 \%$ para la raza Holstein, $32,10 \%$ para la raza Jersey y $30,54 \%$ para los cruces en diferentes proporciones. De los registros evaluados para la variable producción de leche diaria, el 37,29\% correspondió a la raza Holstein, el 32,03\% para el ganado Jersey y de $30,68 \%$ para los cruces.

Análisis de la información: La información fue organizada según un diseño irrestricto al azar, el cual fue analizado con ayuda del programa PROC GLM de SAS (2003), en donde se valoró el efecto que presentaba la raza del animal (Holstein, Jersey y sus cruces en diferentes proporciones), sobre los valores promedio de las variables reproductivas (IEP y DA) y las variables productivas. Cuando se determinaron diferencias significativas entre medias, estas se separaron por medio de la prueba de Duncan con una probabilidad $(p<0,05)$ (SAS, 2003). Se determinó el coeficiente de chance o "Odds Ratio" para definir la posibilidad que presenta cada raza evaluada en obtener un valor mayor en comparación a otra raza, al considerar producción de leche, el intervalo entre parto y el periodo abierto, según los datos obtenidos (Mc Hugh, 2009). Para tal fin, se tomó el valor promedio de toda la población, conformada por la sumatoria de los datos de la raza Jersey, Holstein y sus cruces en diferentes proporciones para definir el punto de separación entre un valor alto a un valor bajo en cada indicador evaluado para cada raza.

\section{RESULTADOS}

Parámetros reproductivos. En el Cuadro 1 se observa el desempeño reproductivo de las razas evaluadas y sus cruces en diferentes proporciones según los datos 
CUADRO 1

Parámetros productivos y reproductivos según la raza Holstein, Jersey y sus cruces

\begin{tabular}{|c|c|c|c|c|c|}
\hline \multirow[b]{2}{*}{ Raza } & \multicolumn{5}{|c|}{ Parámetros técnicos ${ }^{2}$} \\
\hline & $\begin{array}{l}\text { Intervalo entre partos } \\
\text { (Días) }\end{array}$ & $\begin{array}{l}\text { Días abiertos } \\
\text { (Días) }\end{array}$ & $\begin{array}{c}\text { Días en lactancia } \\
\text { (Días) }^{3}\end{array}$ & $\begin{array}{l}\text { Producción láctea } \\
\text { (kg) }\end{array}$ & $\begin{array}{l}\text { Urea en leche } \\
\text { (mg/dl) }\end{array}$ \\
\hline Holstein & $434,49^{a}$ & $141,34^{a}$ & $187,85^{\mathrm{ab}}$ & $23,85^{a}$ & $16,09^{b}$ \\
\hline Dominancia Holstein & $438,81^{a}$ & $146,11^{a}$ & $206,52^{a}$ & $22,36^{\mathrm{ab}}$ & $14,86^{c}$ \\
\hline Holstein X Jersey (F1) & $392,65^{b}$ & $119,16^{b}$ & $169,87^{\mathrm{bc}}$ & $23,78^{a}$ & $14,69^{c}$ \\
\hline Dominancia Jersey & $383,75^{b}$ & $98,53^{c}$ & $148,72^{c}$ & $23,09^{\mathrm{ab}}$ & $14,72^{c}$ \\
\hline Jersey & $404,05^{b}$ & $121,06^{b}$ & $173,85^{b}$ & $22,08^{b}$ & $19,29^{a}$ \\
\hline
\end{tabular}

1. Letras diferentes en una misma columna, presentan diferencias significativas $(p<0,05)$.

2. Datos promedio por animal

3. Días promedio de hato para cada raza.

recolectados y el análisis planteado. Para los parámetros reproductivos, IEP y días abiertos, la raza Holstein superó a la raza Jersey, a los cruces HxJ F1 y a los cruces con dominancia Jersey en 30,44 y 20,$28 ; 41,84$ y 22,$18 ; 50,74$ y 42,81 días respectivamente para cada parámetro $(p<0,05)$. No así, a los cruces HxJ con dominancia Holstein, donde no se determinaron diferencias significativas.

Parámetros productivos. En el caso de la producción diaria de los animales (Cuadro 1), en esta investigación se obtuvo que, la raza Jersey, presento una producción de leche menor en 1,$77 ; 0,28 ; 1,7$ y $1,01 \mathrm{~kg}$ de leche al compararla con la raza Holstein, los cruces $\mathrm{HxJ}$ con predominancia Holstein, animales F1 y cruces con predominancia Jersey respectivamente $(p<0,05)$. En el caso de la raza Holstein y los cruces $\mathrm{HxJ}$ en diferentes proporciones no se determinó diferencia significativa $(p>0,05)$ en la productividad de leche diaria.

En relación a los días en lactancia para cada grupo racial analizado (Cuadro 1), se nota una tendencia en los animales, de reducir el promedio de días en lactancia conforme aumentan la proporción del patrón racial Jersey en el cruce $(p<0,05)$. Según los resultados obtenidos, no se encontraron diferencias significativas entre animales Jersey y Holstein, para este parámetro.

Al analizar el contenido de urea en la leche de los animales, los animales producto de un cruce, presentan diferencias de 1,33 a 4,54mg de urea por cada decilitro de leche $(p<0,05)$ al compararse con sus razas paternas o maternas. Pero al evaluar los animales cruzados, no se determinaron diferencias en la proporción de las razas bases, donde los valores fluctuaron entre 14,69 a 14,86 (mg/dl) $(p>0,05)$.

Asociaciones relativas entre razas para los indicadores de producción de leche diaria, intervalo entre parto y periodo abierto. Se describe en el Cuadro 2, para la variable producción de leche diaria, que la raza Holstein presenta una mayor probabilidad de generar una mayor producción de leche diaria que los animales

\section{CUADRO 2}

Coeficiente de chance e intervalos de confianza al $95 \%$ entre las razas Holstein, Jersey y sus cruces en diferentes proporciones para los indicadores producción de leche diaria, intervalo entre parto y periodo abierto

\begin{tabular}{|c|c|c|c|c|}
\hline \multicolumn{2}{|l|}{ Variables } & $\begin{array}{c}\text { Holstein en comparación } \\
\text { a Jersey }\end{array}$ & $\begin{array}{c}\text { Holstein en comparación } \\
\text { a cruces } \mathrm{HxJ} J^{1 .}\end{array}$ & $\begin{array}{c}\text { Jersey en comparación } \\
\text { a cruces } \mathrm{HxJ}{ }^{1 .}\end{array}$ \\
\hline \multirow[t]{2}{*}{ Producción de leche } & $\mathrm{OR}^{2}$ & 1,65 & 1,03 & 0,62 \\
\hline & IC $95 \%{ }^{3 .}$ & $1,29-2,12$ & $0,80-1,32$ & $0,48-0,81$ \\
\hline \multirow[t]{2}{*}{ Intervalo entre partos } & OR & 1,60 & 2,11 & 1,32 \\
\hline & IC $95 \%$ & $1,19-2,15$ & $1,54-2,89$ & $0,95-1,81$ \\
\hline \multirow{2}{*}{ Periodo abierto } & OR & 1,72 & 2,06 & 1,20 \\
\hline & IC $95 \%$ & $1,31-2,27$ & $1,55-2,74$ & $0,88-1,62$ \\
\hline
\end{tabular}

1. Cruces Holstein $x$ Jersey en diferentes proporciones.

2. Odds Ration.

3. Intervalo de confianza. 
Jersey y que los cruces en diferentes proporciones. Si es importante considerar el intervalo de confianza, obtenido en la comparación de la raza pura y los cruces, debido a que el rango al estar por debajo de cero, indica que ambos grupos podrían presentar niveles altos de producción. En relación a las variables reproductivas (Cuadro 2), los valores del coeficiente de chance sugieren que los animales Holstein, son propensos a presentar mayor cantidad de días entre cada parto y para iniciar una nueva gestación. De igual manera, como se presentó en la variable producción de leche diaria, la probabilidad de presentar menor cantidad de días para el IEP y el PA, es la misma al comparar el animal Jersey y los cruces, debido al valor obtenido del intervalo de confianza.

\section{DISCUSIÓN}

En cuanto a la raza Jersey, los trabajos de Urbano, Rodríguez-Voigt, Dávila-Calderón, Verde, Carroz, Rodríguez y Berbin (2000) y Echeverri et al. (2011) documentan al analizar la información de grupos de animales, el buen desempeño en el ámbito reproductivo de esta raza, al informar valores promedio de 371 y 417 días de IEP por ciclos productivos respectivamente. En donde lo relacionan a su bajo peso corporal y a la alta capacidad de conversión de alimento, lo cual las hace animales mejor alimentadas por su bajo requerimiento de consumo, por ende, con mejor condición corporal y a la vez de salud para su reproducción. En el caso de los animales producto al cruce HxJ, Urbano et al. (2000) y Echeverri et al. (2011) aseveran que el efecto de vigor hibrido podría ser el responsable de que estos animales presenten mejores indicadores, en condiciones similares de manejo y ambientales. Relacionado a esta mejora en la interacción de los genes con el ambiente, en trabajos realizados por Heins et al. (2008) indican que los animales provenientes del cruce Holstein y Jersey presentan similitud en el consumo de materia seca y el consumo de materia seca según proporción a su peso corporal, lo que permite, que ambos sistemas ruminales en condiciones similares de manejo, presente los mismos aporte de carbohidratos, minerales y proteína, necesarios para satisfacer los requerimientos de proteína y energía. Estos mismos investigadores demostraron que el cruce $\mathrm{HxJ}$, presentó un menor peso (-33 kg/pv) y una calificación de condición corporal mayor $(+0,14)$, situación que permite a los animales cruzados, el requerimiento de mantenimiento sea menor, y se pueda utilizar los nutrimentos consumidos en otras funciones. Para la raza Holstein, los datos obtenidos en esta investigación podrían estar relacionados a los problemas de enfermedades, asociadas al periodo de transición del periodo seco al de lactación temprana por desbalances metabólicos, respaldado por Uribe, Kennedy, Martin y Kelton (1995) que habla de lo propenso de la raza Holstein por su alta producción y la tendencia a heredar ciertas enfermedades de lactancia temprana. Estas enfermedades en trabajos de Mulligan, Grady, Rice y Doherty (2006), tiene un parámetro máximo aceptable en hatos lecheros de: cetosis 5\%, desplazamiento de abomaso $4 \%$, fiebre de leche $5 \%$, mastitis $3 \%$, metritis $10 \%$ y retención de placenta $8 \%$. En este mismo tema, Curtis, Erb, Sniffen, Smith y Kronfeld (1985) indicaron que la incidencia de enfermedades en el periodo de transición genera un impacto en la probabilidad de concepción y así el aumento de los días abiertos. Los diferentes autores concuerdan con lo obtenido para los indicadores reproductivos de los animales incluidos en esta investigación, donde las razas Jersey y los cruces $\mathrm{HxJ}$ en diferentes proporciones aventajan a los animales de la raza Holstein, siendo animales eficientes en sus parámetros reproductivos, lo que permite obtener hatos con una reproducción estable, en el cual los animales tendrán mayor cantidad de partos en su vida productiva en un lapso de menor de tiempo. Al comparar los datos de esta investigación con los resultados descritos por González y Boschini (1995) se obtiene una tendencia similar, donde la raza Holstein supera a la raza Jersey en producción diaria. De igual manera Vargas, Marin y Romer (2012)., obtienen que animales de la raza Holstein superan a los animales del cruce $\mathrm{HxJ}$ en diferentes proporciones y a la raza Jersey en producción de leche al año. No así en el contenido de sólidos lácteos, donde el cruce $\mathrm{HxJ}$ en diferentes proporciones superó a la raza Holstein y la raza Jersey (Vargas et al., 2012).

En cuanto a los días de lactancia promedio del hato, se determinó que la raza Holstein presentó la mayor cantidad de días en promedio $(p<0,05)$, al compararse con la raza Jersey y los cruces $\mathrm{HxJ}$ en diferentes proporciones. Vargas et al. (2012) informan que este comportamiento de la raza Holstein se podría ligar a una mayor cantidad de días entre el parto y el inicio de la siguiente gestación, lo que provoca que estos animales se mantengan más días en lactancia por no estar preñadas, lo que se comprueba en esta investigación al analizar el IEP, donde se observa que al ser más prolongados en la raza Holstein, es indicativo de que estos animales se mantienen más tiempo en producción (Cuadro 1). Según el promedio de días de lactancia determinados en esta investigación para la raza Jersey y los cruces $\mathrm{HxJ}$ en diferentes proporciones, estos valores se encuentran cercanos al valor ideal de 160 días de lactancia promedio, que debería tener un hato en producción, en donde Hernández, Ortega, Fernández, Raigoza y Montaldo (2006) manifiesta que se 
obtiene un balance ideal entre los animales que paren y los que se tienen que secar, lo cual permite mantener una estabilidad de la productividad del hato a lo largo del año. Todos estos datos hacen evaluar la escogencia de la raza de las vacas o analizar el manejo que se le da al hato a la hora de tomar una decisión que pueda repercutir en los valores financieros de la lechería.

En el caso de los animales Holstein, el mayor intervalo entre parto y días abiertos se relaciona a un proceso de mejoramiento genético en esta raza, que generó animales más productivos, pero de forma paralela, seleccionó animales menos fértiles (Hernández et al., 2006). Este aumento en los días para estas variables se traduce en ineficiencia y reducción en la rentabilidad del sistema, la cual se relaciona a menor producción de leche e incremento en el costo del manejo reproductivo (HosseinZadeh, 2013).

Según los resultados obtenidos, queda claro, la mejora en los parámetros reproductivos, de los animales obtenidos del cruce de los animales Holstein y Jersey en diferentes proporciones, lo cual refleja una mayor vida productiva de los semovientes en el sistema. En el caso de los aspectos productivos, se obtiene una producción de leche mayor en la raza Holsteins y sus cruces que los animales Jersey, esta diferencia ponderada $(1,19 \mathrm{~L}$ de leche/animal/día), hace que, en lactancias ajustadas a 305 días, los animales con un patrón racial Holstein produzcan $362,99 \mathrm{~L}$ de leche por lactancia. Consideraciones importantes a la hora de seleccionar el tipo de semoviente a mantenerse en el sistema de producción.

\section{AGRADECIMIENTOS}

Los autores agradecen al Comité de Educación y Bienestar Social de la Cooperativa de Productores de Leche R.L. por el apoyo brindado en el desarrollo de esta investigación

\section{REFERENCIAS}

Aamoun, A. (2015). Water footprint of cow milk production (Bachelor's Thesis). NOVIA, University of Applied Sciense, Raseborg.

Bačić, G., Karadjole, T. , Mačešić, N., \& Karadjole, M. (2007). A brief review of etiology and nutritional prevention of metabolic disorders in dairy cattle. Veterinarski arhiv, 77(6), 567-577.

Buckley, F., Lopez-Villalobos, N., \& Heins, B.J. (2014). Crossbreding: implications for dairy cow fertility and survival. Animal, 8(s1),122-133 p.
Cordero-Solórzano, J. M., Vargas-Leitón, B., León-Rodríguez, B., Chacón-González, I., \& Martínez-Pichardo, M. (2015). Diversidad genetic en bovines de ocho regiones en Costa Rica. Agronomía Mesoamericana, 26(2), 191-202. doi:10.15517/am.v26i2.19275

Curtis, C.; Erb, H., Sniffen, C.; Smith, R., \& Kronfeld, D. (1985). Path analysis of dry period nutrition, postpartum metabolic and reproductive disorders, and mastitis in Holstein cows. Journal of dairy science, 68(9), 2347-2360. doi:10.3168/jds.S0022-0302(85)81109-7

Echeverri, J., Salazar, V., \& Parra, J. (2011). Análisis comparativo de los grupos genéticos Holstein, Jersey y algunos de sus cruces en un hato lechero del norte de Antioquia en Colombia. Zootecnia Tropical, 29(1), 49-59

Heins, B., Hansen, L., Seykora, A., Johnson, D., Linn, J., Romano, J., \& Hazel, A. (2008). Crossbreds of JerseyXHolstein compared with pure Holsteins for production, fertility, and body and udder measurements during first lactation. . Journal of dairy science, 91, 1270-1278. doi: 10.3168/ jds.2007-0564

Herburt, P. \& Angrecka S. (2017). Relationship between THI level and dairy cow's behavior durin summer period. Italian Journal of Animal Science, 17(1), 226-233. doi: 10.1080/1828051X.2017.1333892

Hernández, J., Ortega, A, Fernández, I., Raigoza, G., \& Montaldo, H. (2006). Fertilidad y producción de leche de vacas Holstein americanas, australianas y uruguayas en estabulado. Archivos de Zootecnia, 55 (211), 289-292.

Hossein-Zadeh, G. (2013). Effects of main reproductive and health problems on the performance of dairy cows: review. Spanish Journal of Agriculture Research, 11(3), 718735. doi: 10.5424/sjar/2013113-4140

INEC (Instituto Nacional de Censos, Costa Rica). (2015). VI Censo Nacional Agropecuario: Características de las Fincas y de las personas productoras. Resultados generales. Instituto Nacional de Estadísticas y Censos -1 ed-San José, Costa Rica. 178 p.

González, N., \& Boschini, C. (1995). Comportamiento de la producción de leche en hatos Holstein y Jersey del Valle Central de Costa Rica. Nutrición Animal Tropical, 2(1), 1409-3553.

McAllister, A. J. (2002). Is Crossbreeding the answer to questions of dairy breed utilization? Journal of dairy science, 85, 2352-2357. doi:10.3168/jds.S0022-0302(02)74315-4

McHugh, M. (2009). The odds ratio: calculation, usage, and interpretation. Biochemia Medica, 19(2), 120-6. doi: 10.11613/BM.2009.011

Mulligan, F., Grady, L., Rice, D., \& Doherty, M. 2006. A herd health approach to dairy cow nutrition and production diseases of the transition cow. Animal Reproduction Science, 96(3-4), 331-353. doi: 10.1016/j.anireprosci.2006.08.011

Rotz, CA., Montes, F. \& Chianese S. (2010). The carbón footprint of dairy productions systems through partial life cycle 
assessement. Journal of dairy science, 93, 1266-1282. doi: 10.3168/jds.2009-2162

SAS (SAS Institute Inc. US). (2003). SAS 9.1.3 for Windows. Service Pack 4. Win_Pro plataforma. Copyright (C) 2002-2003 Cary NC.

Urbano, D., Rodríguez-Voigt, A., Dávila-Calderon, C., Verde, O, Carroz, R., Rodríguez, M., \& Berbin, W. (2000). Comportamiento de hembras Holstein, Jersey y mestizas en el Estado Mérida. Fondo Nacional de Investigacion Agropecuaria, Maracay (Venezuela). FONAIAP, 67, 35-37.

Uribe, H., Kennedy, B., Martin, S., \& Kelton, D. (1995). Genetic parameters for common health disorders of Holstein cows. Journal of Dairy Science, 78(2), 421-430.

VanRaden, P. M., \& Sanders, A. H. (2003). Economic merit of crossbred and purebed US dairy cattle. Journal of Dairy Science, 86, 1036-1044. doi: 10.3168/jds. S0022-0302(03)73687-X
Vargas, B., Marin, Y., \& Romer, J. (2012). Comparación bioeconómica de grupos raciales Holstein, Jersey y HolsteinXJersey en Costa Rica. Agronomía Mesoamericana, 23(2), 329342. doi: 10.15517/am.v23i2.6533

Vargas-Leitón, B., Solís-Guzmán, O., Sáenz-Segura, F., \& LeónHidalgo, H. (2015). Eficiencia técnica en hatos lecheros de Costa Rica. Agronomía Mesoamericana 26(1), 1-15. doi: 10.15517/am.v26i1.16886

Vargas-Leitón, B., \& Romero-Zúñiga, J. (2010). Efectos genéticos aditivos y no aditivos en cruces rotacionales entre razas lecheras. Agronomía Mesoamericana, 21, 223-234. doi:10.15517/am.v21i2.4885

Vásquez, J. F., Barrios, D., \& Olivera, M. (2015). Análisis del desempeño técnico económico de un hato lechero en Colombia: estudio de caso. Revista Veterinaria y Zootecnia, 9(1), x-x doi: 10.17151/vetzo.2015.9.1.7. 